\title{
Interaction-induced correlations and non-Markovianity of quantum dynamics
}

\author{
A. Smirne, ${ }^{1}$ L. Mazzola,${ }^{2}$ M. Paternostro, ${ }^{2}$ and B. Vacchini ${ }^{1}$ \\ ${ }^{1}$ Dipartimento di Fisica, Università degli Studi di Milano, Via Celoria 16, I-20133 Milan, Italy and INFN, Sezione di Milano, \\ Via Celoria 16, I-20133 Milan, Italy \\ ${ }^{2}$ Centre for Theoretical Atomic, Molecular and Optical Physics, School of Mathematics and Physics, Queen's University, \\ Belfast BT7 1NN, United Kingdom
}

(Received 7 February 2013; published 22 May 2013)

\begin{abstract}
We investigate the conditions under which the trace distance between two different states of a given open system increases in time due to the interaction with an environment, therefore signaling non-Markovianity. We find that the finite-time difference in trace distance is bounded by two sharply defined quantities that are strictly linked to the occurrence of system-environment correlations created throughout their interaction and affecting the subsequent evolution of the system. This allows us to shed light on the origin of non-Markovian behaviors in quantum dynamics. We best illustrate our findings by tackling two physically relevant examples: a non-Markovian dephasing mechanism that has been the focus of a recent experimental endeavor and the open-system dynamics experienced by a spin connected to a finite-size quantum spin chain.
\end{abstract}

DOI: 10.1103/PhysRevA.87.052129

PACS number(s): 03.65.Yz, 03.65.Ta, 42.50.Lc

\section{INTRODUCTION}

Recently great attention has been paid to the development of a more general understanding of the dynamics of open quantum systems, in order to deal with the occurrence of memory effects [1-18]. In particular, different definitions of quantum non-Markovianity have been theoretically introduced [19-22] and, in some cases, experimentally investigated [23-26]. Nevertheless, the physical reasons ruling whether an open quantum system exhibits a Markovian or a non-Markovian dynamics have still to be fully clarified.

A widely accepted view is that, in Markovian dynamics, the correlations between the open system and its environment as well as the changes in the environmental state due to the interaction do not have a significant influence on the subsequent evolution of the open system. This picture is often introduced relying on qualitative considerations, possibly assuming that the total state at time $t$ can be effectively represented as a product state between the state of the open system at the time $t$ and a fixed state of the environment $[1,27]$. It is worth noting how the same assumption also lies at the foundations of the quantum regression hypothesis [28-31]. System-environment correlations induced by the interaction and changes in the state of the environment are thus thought to be at the basis of non-Markovian dynamics.

In this paper, we show how this relationship can be formulated in a quantitative way using the properties of the trace distance [32], whose time evolution can be used to characterize the dynamics of an open quantum system that evolves starting from two different initial states [20,33,34]. Any change of the trace distance between states of an open system can be interpreted as an exchange of information with the environment that affects it: a nonmonotonic behavior of the trace distance witnesses the fact that some information previously lost by the open system can affect it back again, thus inducing memory effects in its evolution. In view of this interpretation, non-Markovian dynamics can be identified with those dynamics that show an increase of the trace distance at some intervals of time [20,34].
Here, starting from the analysis reported in Ref. [16], we introduce an upper and a lower bound to the variation of the trace distance at finite time intervals. The bounds express quantitatively the influence of the system-environment correlations and the changes in the state of the environment at a time $t$ on the subsequent dynamics of the open system. They thus allow an estimation of how system-environment correlations, as well as evolution of the environment, account for the Markovian or non-Markovian nature of an open system's dynamics. In particular our lower bound provides a sufficient condition for the onset of non-Markovianity. We apply our analysis to two physical examples: the experimental setting considered in Ref. [23] that describes the transition from Markovian to nonMarkovian dephasing on a qubit and the energy-nonconserving open-system dynamics of a spin-1/2 particle that is coupled to a finite-size quantum spin chain $[9,35]$.

The remainder of this paper is organized as follows. In Sec. II, we first introduce an upper and a lower bound to the variation of the trace distance, which hold under very general conditions. We further show how, as a consequence, the strength of the effects of system-environment correlations and environmental evolution can determine the non-Markovian character of a given dynamics. In Sec. III, we apply our general analysis to two physically relevant examples. First, we address the single-qubit pure-dephasing mechanism exploited in Ref. [23] to investigate experimentally the transition between Markovian and non-Markovian dynamics. Second, we study the case of a single spin interacting with a finite-size spin environment embodied by a quantum spin chain $[9,35]$. Finally Sec. IV is devoted to conclusions and final remarks.

\section{ROLE OF SYSTEM-ENVIRONMENT CORRELATIONS AND ENVIRONMENTAL EVOLUTION IN THE DYNAMICS OF OPEN QUANTUM SYSTEMS}

\section{A. Upper and lower bounds to the increase of the trace distance on a finite time interval}

We now highlight the relevance of the correlations between system and environment, as well as of the changes in the state 
of the environment, in determining the variation of the trace distance among system states. As discussed above, knowledge of the time dependence of this quantity allows an assessment of the Markovianity properties of the time evolution, and is therefore of primary importance. With this aim let us start from the decomposition of any joint system-environment state $\rho_{S E}$ as [36]

$$
\rho_{S E}=\rho_{S} \otimes \rho_{E}+\chi_{S E},
$$

where $\rho_{S}\left(\rho_{E}\right)$ is the reduced state of the system $S$ (the environment $E$ ) and $\chi_{S E}\left(\right.$ such that $\left.\operatorname{tr}_{E}\left[\chi_{S E}\right]=\operatorname{tr}_{S}\left[\chi_{S E}\right]=0\right)$ accounts for the total correlations between the open system and the environment, as also shown by the relation $\left\|\chi_{S E}\right\|=$ $2 D\left(\rho_{S} \otimes \rho_{E}, \rho_{S E}\right)$. The total $S-E$ system is usually assumed to be closed, so that its evolution is provided by a one-parameter group of unitary operators $\left\{U_{t}\right\}_{t \geqslant 0}$, with $t_{0}=0$ the initial time. Given the initial total state $\rho_{S E}(0)$, the total state at a time $t$ is $\rho_{S E}(t)=U_{t} \rho_{S E}(0) U_{t}^{\dagger}$ and, as a consequence, the state at time $t+t^{\prime}$ can be inferred from the state at the time $t$ through the relation

$$
\rho_{S E}\left(t+t^{\prime}\right)=U_{t^{\prime}, t} \rho_{S E}(t) U_{t^{\prime}, t}^{\dagger},
$$

where $U_{t^{\prime}, t}=U_{t+t^{\prime}} U_{t}^{\dagger}$.

Using Eq. (1) twice, we can simply express the difference in the total states at time $t$ originating from different initial conditions as follows:

$$
\begin{aligned}
& \rho_{S E}^{1}(t)-\rho_{S E}^{2}(t) \\
& =\left[\rho_{S}^{1}(t)-\rho_{S}^{2}(t)\right] \otimes \rho_{E}^{1}(t) \\
& \quad+\rho_{S}^{2}(t) \otimes\left[\rho_{E}^{1}(t)-\rho_{E}^{2}(t)\right]+\left[\chi_{S E}^{1}(t)-\chi_{S E}^{2}(t)\right] .
\end{aligned}
$$

An equivalent relation is obtained by exchanging the roles of labels 1 and 2 . The difference between the total states can thus be split into two contributions, one depending on the difference between the states of the reduced systems and the other, made up of the last two contributions on the right-hand side (RHS) of Eq. (3), given by the comparison between the reduced environmental states and between the correlations. We thus identify the two quantities

$$
\begin{aligned}
F\left(t^{\prime}, t, \rho^{1,2}\right) \equiv & D\left(\operatorname{tr}_{E}\left[U_{t^{\prime}, t}\left(\rho_{S}^{1}(t) \otimes \rho_{E}^{1}(t)\right) U_{t^{\prime}, t}^{\dagger}\right],\right. \\
& \left.\operatorname{tr}_{E}\left[U_{t^{\prime}, t}\left(\rho_{S}^{2}(t) \otimes \rho_{E}^{1}(t)\right) U_{t^{\prime}, t}^{\dagger}\right]\right)
\end{aligned}
$$

and

$$
\begin{aligned}
B\left(t^{\prime}, t, \rho^{1,2}\right) \equiv & \frac{1}{2} \| \operatorname{tr}_{E}\left[U_{t^{\prime}, t}\left(\rho_{S}^{2}(t) \otimes\left[\rho_{E}^{1}(t)-\rho_{E}^{2}(t)\right]\right) U_{t^{\prime}, t}^{\dagger}\right] \\
& +\operatorname{tr}_{E}\left[U_{t^{\prime}, t}\left(\chi_{S E}^{1}(t)-\chi_{S E}^{2}(t)\right) U_{t^{\prime}, t}^{\dagger}\right] \|,
\end{aligned}
$$

where we have introduced the trace norm of an element $\sigma$ of the set $\mathcal{T}$ of linear trace class operators as $\|\sigma\|=\operatorname{Tr}\left[\sqrt{\sigma^{\dagger} \sigma}\right]$ and the trace distance between two statistical operators $\rho^{1,2}$,

$$
D\left(\rho^{1}, \rho^{2}\right)=\frac{1}{2}\left\|\rho^{1}-\rho^{2}\right\|=\frac{1}{2} \sum_{k}\left|\varrho_{k}\right|,
$$

with $\varrho_{k}$ the eigenvalues of the self-adjoint traceless operator $\rho^{1}-\rho^{2}$ and $0 \leqslant D\left(\rho^{1}, \rho^{2}\right) \leqslant 1 . D\left(\rho^{1}, \rho^{2}\right)$ quantifies the distinguishability [32] between two given states. If a system is prepared in one of the two states $\rho^{1}$ or $\rho^{2}$ with probability $1 / 2$ each, an observer can design an optimal strategy to guess the preparation with success probability given by $\left[1+D\left(\rho^{1}, \rho^{2}\right)\right] / 2$. Note that the two quantities defined in Eqs. (4) and (5) depend on the times $t$ and $t^{\prime}$, as well as on the two initial states $\rho_{S E}^{1,2}(0)$.

The quantity $F\left(t^{\prime}, t, \rho^{1,2}\right)$ describes how the distinguishability between the reduced states would evolve at a time $t+t^{\prime}$ if the two total states at the time $t$ were product states, with the same environmental state, thus building on the first term on the RHS of Eq. (3). In a complementary way, the quantity $B\left(t^{\prime}, t, \rho^{1,2}\right)$ keeps track of the effects of correlations and differences in the environmental states at a time $t$ on the subsequent dynamics of the open system. Thanks to the contractivity of the trace norm of a self-adjoint operator under the action of any completely positive trace-preserving linear (CPT) map [37], it is straightforward to show that $B\left(t^{\prime}, t, \rho^{1,2}\right) \in[0,2]$. Moreover, it can be null even if the total states at time $t$ are not product states, so that the correlations, despite being present, do not have any influence on the evolution of the trace distance between two reduced states of $S$.

In what follows, we will study the evolution of the distinguishability between pairs of reduced states, thus describing the information flow between the open system and its environment $[20,33,34]$. The trace distance between two reduced states at time $t$ will be indicated as

$$
D\left(t, \rho^{1,2}\right) \equiv D\left(\rho_{S}^{1}(t), \rho_{S}^{2}(t)\right),
$$

where $\rho_{S}^{j}(t)=\operatorname{tr}_{E}\left[U_{t} \rho_{S E}^{j}(0) U_{t}^{\dagger}\right], j=1,2$. Our analysis focuses on the variation of the trace distance at finite time intervals

$$
\Delta D\left(t^{\prime}, t, \rho^{1,2}\right) \equiv D\left(t+t^{\prime}, \rho^{1,2}\right)-D\left(t, \rho^{1,2}\right) .
$$

Using Eqs. (2) and (3), the trace distance between two reduced states at time $t+t^{\prime}$ can be expressed as the sum of two different contributions, which reflect the decomposition at the RHS of Eq. (3). Then the triangular inequalities for the trace norm,

$\left|\|\sigma\|-\left\|\sigma^{\prime}\right\|\right| \leqslant\left\|\sigma-\sigma^{\prime}\right\| \leqslant\|\sigma\|+\left\|\sigma^{\prime}\right\| \forall \sigma, \sigma^{\prime} \in \mathcal{T}$,

directly lead to the following bounds to the variation of the trace distance on finite time intervals:

$$
B\left(t^{\prime}, t, \rho^{1,2}\right)-F\left(t^{\prime}, t, \rho^{1,2}\right)-D\left(t, \rho^{1,2}\right) \leqslant \Delta D\left(t^{\prime}, t, \rho^{1,2}\right) \leqslant B\left(t^{\prime}, t, \rho^{1,2}\right)+F\left(t^{\prime}, t, \rho^{1,2}\right)-D\left(t, \rho^{1,2}\right) .
$$

Equation (10) holds in complete generality, the only requirement being that one takes into account the full unitary evolution $U_{t}$, which could well be time inhomogeneous. The upper bound in Eq. (10) shows that

$$
B\left(t^{\prime}, t, \rho^{1,2}\right)>D\left(t, \rho^{1,2}\right)-F\left(t^{\prime}, t, \rho^{1,2}\right)
$$


is a necessary condition for the increase of the trace distance within the time interval $\left[t, t+t^{\prime}\right]$. It is important to note that the RHS of Eq. (11) is positive as a consequence of the contractivity of the trace norm. In fact, $F\left(t^{\prime}, t, \rho^{1,2}\right)$ can be written as [16]

$$
F\left(t^{\prime}, t, \rho^{1,2}\right)=D\left(\Phi_{t^{\prime}, t}\left[\rho_{S}^{1}(t)\right], \Phi_{t^{\prime}, t}\left[\rho_{S}^{2}(t)\right]\right),
$$

where

$$
\Phi_{t^{\prime}, t}\left[\rho_{S}\right]=\operatorname{tr}_{E}\left[U_{t^{\prime}, t}\left(\rho_{S} \otimes \rho_{E}^{1}(t)\right) U_{t^{\prime}, t}^{\dagger}\right]
$$

for any $t$ and $t^{\prime}$, so that $\Phi_{t^{\prime}, t}$ is a CPT map [1] and $F\left(t^{\prime}, t, \rho^{1,2}\right) \leqslant D\left(t, \rho^{1,2}\right)$, with $F\left(0, t, \rho^{1,2}\right)=D\left(t, \rho^{1,2}\right)$. An increase of the distinguishability between reduced states needs the effects of system-environment correlations to prevail over the contraction of the trace distance due to the reduced CPT map $\Phi_{t^{\prime}, t}$ obtained from a total product state at time $t$. Notice that in the limit $t^{\prime} \rightarrow 0$ the upper bound in Eq. (10) leads to the bound found in Ref. [16]. Finally, by applying again the triangular inequality and using the contractivity of the trace norm under CPT maps on Eq. (10), one gets the weaker upper bound

$$
\begin{aligned}
\Delta D\left(t^{\prime}, t, \rho^{1,2}\right) \leqslant & D\left(\rho_{S E}^{1}(t), \rho_{S}^{1}(t) \otimes \rho_{E}^{1}(t)\right) \\
& +D\left(\rho_{S E}^{2}(t), \rho_{S}^{2}(t) \otimes \rho_{E}^{2}(t)\right) \\
& +D\left(\rho_{E}^{1}(t), \rho_{E}^{2}(t)\right) .
\end{aligned}
$$

This confirms that an increase of the trace distance in the time interval $\left[t+t^{\prime}, t\right]$ calls for system-environment correlations in at least one of the two total states at time $t$, or for different environmental states $\rho_{E}^{1}(t)$ and $\rho_{E}^{2}(t)$. The inequality in Eq. (14) was first derived in Ref. [33] taking $t=0$ as the initial time of the dynamics and pointing out that an increase of the trace distance above its initial value witnesses initial correlations or different initial environmental states. Indeed, a nonmonotonic temporal behavior of the trace distance can be read as an increase with respect to its value at a previous time.

Up to now we have seen how system-environment correlations can be in general compatible also with a decrease of the distinguishability between reduced states. But to what extent is this the case? Can the effects of correlations and environmental evolution be arbitrarily strong without inducing any tracedistance increase? This question is answered by virtue of the lower bound in Eq. (10). A sufficient condition to have an increase of the trace distance in the time interval $\left[t, t+t^{\prime}\right]$ is that the effects at the time $t+t^{\prime}$ of the system-environment correlations and environmental states at the time $t$ are strong enough to satisfy

$$
B\left(t^{\prime}, t, \rho^{1,2}\right)>D\left(t, \rho^{1,2}\right)+F\left(t^{\prime}, t, \rho^{1,2}\right) .
$$

Let us emphasize how the fulfillment of this condition implies the occurrence, with certainty, of an increase of the trace distance, which is quite a remarkable result.

\section{B. System-environment correlations and quantum non-Markovianity}

In virtue of the bounds to the variation of the trace distance discussed in the previous section, we can now show that system-environment correlations and changes in the environmental states actually determine the Markovian or non-Markovian character of a dynamics according to the definition given in Ref. [20].

Let us assume a product initial state $\rho_{S E}(0)=\rho_{S}(0) \otimes$ $\rho_{E}(0)$ with a set environmental state $\rho_{E}(0)$, which implies the existence of a well-defined reduced dynamics on the whole set of statistical operators of the open system. In fact, the open system's dynamics can then be described via a family of CPT maps $\{\Lambda(t)\}_{t \geqslant 0}$, with [1]

$$
\Lambda(t)\left[\rho_{S}\right]=\operatorname{tr}_{E}\left[U_{t}\left(\rho_{S} \otimes \rho_{E}^{1}(0)\right) U_{t}^{\dagger}\right]
$$

so that $\rho_{S}(t)=\Lambda(t) \rho_{S}(0)$. Non-Markovianity of quantum dynamics has been defined and quantified in terms of the various properties of this family of CPT maps. In particular, the measure of non-Markovianity $\mathcal{N}(\Lambda)$ introduced in Ref. [20] can be expressed as

$$
\mathcal{N}(\Lambda)=\max _{\rho^{1,2}} \sum_{k} \Delta D\left(b_{k}-a_{k}, a_{k}, \rho^{1,2}\right),
$$

where $\left(a_{k}, b_{k}\right)$ are the time intervals where the trace distance $D\left(t, \rho^{1,2}\right)$ increases, and the maximum over all pairs of initial reduced states is taken. $\mathcal{N}(\Lambda)$ quantifies the total amount of information that flows to the open system, as witnessed by the trace distance, and the relevance of memory effects on the reduced dynamics. From Eq. (17), it is clear that a quantum dynamics is non-Markovian if and only if there is a time interval $\left[t, t+t^{\prime}\right]$ and a pair of initial states $\rho_{S}^{1,2}(0)$ of the system such that $\Delta D\left(t^{\prime}, t, \rho^{1,2}\right)>0$. As we have assumed an initial total product state, with fixed environmental state, the dependence on $\rho^{1,2}$ should be intended from now on as referred to the two initial reduced states. In addition, possible system-environment correlations in the total states, as well as differences between the environmental states, at a time $t$ have entirely to be ascribed to the system-environment interaction up to time $t$.

Equations (11) and (15) provide a general reference scale that relates the Markovian or non-Markovian nature of a given dynamics to the relevance of the quantity $B\left(t^{\prime}, t, \rho^{1,2}\right)$ with respect to $D\left(t, \rho^{1,2}\right)$ and $F\left(t^{\prime}, t, \rho^{1,2}\right)$. This relation is schematically depicted in Fig. 1 , where the horizontal axis represents the possible values of $B\left(t^{\prime}, t, \rho^{1,2}\right)$ together with the

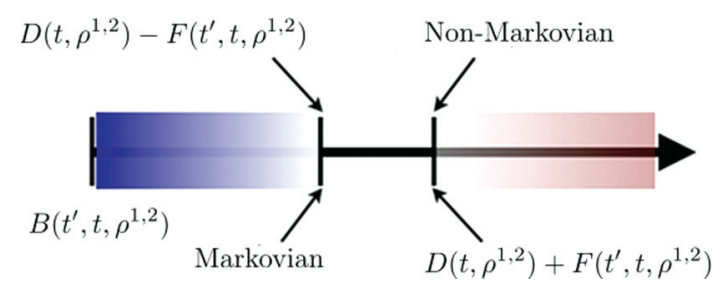

FIG. 1. (Color online) The horizontal axis represents the possible values of $B$ defined in Eq. (5) at fixed times $t$ and $t^{\prime}$ and initial reduced states $\rho_{S}^{1}(0)$ and $\rho_{S}^{2}(0)$, while the two marks represent the thresholds given by $D\left(t, \rho^{1,2}\right)-F\left(t^{\prime}, t, \rho^{1,2}\right)$ (blue region, lower mark) and $D\left(t, \rho^{1,2}\right)+F\left(t^{\prime}, t, \rho^{1,2}\right)$ (red region, upper mark), with $D\left(t, \rho^{1,2}\right)$ and $F\left(t^{\prime}, t, \rho^{1,2}\right)$ defined in Eqs. (7) and (4), respectively. If $B\left(t^{\prime}, t, \rho^{1,2}\right)$ lies above the upper threshold for some $t, t^{\prime}, \rho_{S}^{1,2}(0)$, the dynamics is non-Markovian, while if it takes values below the lower threshold for all the $t, t^{\prime}, \rho_{S}^{1,2}(0)$, the dynamics is Markovian. Values of $B\left(t^{\prime}, t, \rho^{1,2}\right)$ between the two thresholds are compatible with both Markovian and non-Markovian dynamics. 
two reference values given by $D\left(t, \rho^{1,2}\right) \pm F\left(t^{\prime}, t, \rho^{1,2}\right)$. The line above (below) the upper (lower) threshold denotes the region where non-Markovianity (Markovianity) is enforced. If the values of $B\left(t^{\prime}, t, \rho^{1,2}\right)$ are below the lower threshold for any $t, t^{\prime}, \rho_{S}^{1}(0)$, and $\rho_{S}^{2}(0)$, the dynamics is certainly Markovian. On the other hand, the existence of at least one choice of $t, t^{\prime}, \rho_{S}^{1}(0)$, and $\rho_{S}^{2}(0)$ such that $B\left(t^{\prime}, t, \rho^{1,2}\right)$ lies above the upper threshold is enough to induce a non-Markovian dynamics. Finally, the presence of values of $B\left(t^{\prime}, t, \rho^{1,2}\right)$ in the intermediate region of width $2 F\left(t^{\prime}, t, \rho^{1,2}\right)$ does not allow any general statement. Summarizing, even if system-environment correlations and evolution of the environment due to the interaction affect the dynamics of the open system, this does not guarantee that the dynamics is non-Markovian. But the reduced dynamics is surely non-Markovian if the effects of system-environment correlations and environmental evolution, as quantified by $B\left(t^{\prime}, t, \rho^{1,2}\right)$, are strong enough to exceed the upper threshold in Fig. 1.

In addition, $B\left(t^{\prime}, t, \rho^{1,2}\right)$ gives an indication of the degree of non-Markovianity of the dynamics, as expressed by the measure $\mathcal{N}(\Lambda)$ in Eq. (17). Any variation of the trace distance on a finite time interval can be lower bounded through Eq. (10), so that one can introduce a lower bound to $\mathcal{N}(\Lambda)$ via the inequalities

$$
\begin{aligned}
\mathcal{N}(\Lambda) & \geqslant \Delta D\left(t^{\prime}, t, \rho^{1,2}\right) \\
& \geqslant B\left(t^{\prime}, t, \rho^{1,2}\right)-F\left(t^{\prime}, t, \rho^{1,2}\right)-D\left(t, \rho^{1,2}\right),
\end{aligned}
$$

which hold for any $t, t^{\prime}, \rho_{S}^{1}(0)$, and $\rho_{S}^{2}(0)$. Equation (18) implies that $\mathcal{N}(\Lambda)$ is larger the more the effects of systemenvironment correlations and environmental evolution rise above the upper threshold in Fig. 1.

As a further remark, let us notice that our analysis relates the (non-)Markovianity of a given evolution with the general correlations between the system and its environment, regardless of their (quantum or classical) nature. The relevant quantity $B\left(t^{\prime}, t, \rho^{1,2}\right)$ is defined in terms of the total correlations $\chi_{S E}^{j}(t)$, which include both classical and quantum correlations. In general, the interaction does not have to build up quantum correlations in order to determine a reduced non-Markovian dynamics [38]. Indeed, the influence of the quantum or classical nature of system-environment correlations on the trace-distance evolution and, more generally, on the dynamics of open quantum systems is an important issue, still subject to a vivid debate $[5,39,40]$.

\section{EXAMPLES}

\section{A. Transition from Markovian to non-Markovian dephasing}

We now consider, as our first explicit example, the model described in Ref. [23], where the transition from Markovian to non-Markovian dephasing dynamics has been experimentally realized by modifying the initial state of the environment. The total system under investigation consists of single photons generated by spontaneous parametric down-conversion and passing through a Fabry-Pérot cavity mounted on a rotor and then through a quartz plate. The open-system qubit is encoded in the space spanned by two orthogonal polarization states, while the frequency of the photons is used to encode the environment. The dephasing dynamics experienced by the polarization of light and due to the quartz plate can be described through the unitary system-environment evolution

$$
U(t)|\lambda, \omega\rangle=e^{i n_{\lambda} \omega t}|\lambda, \omega\rangle \quad(\lambda=H, V),
$$

where $|H\rangle$ and $|V\rangle$ denote horizontal and vertical polarization states, $n_{\lambda}$ is the refractive index of the plate for a $\lambda$-polarized incident photon, and $|\omega\rangle$ stands for the environmental state at set frequency $\omega$. The initial state of the environment is $\rho_{E}(0)=\left|\psi_{E}(0)\right\rangle\left\langle\psi_{E}(0)\right|$ with

$$
\left|\psi_{E}(0)\right\rangle=\int d \omega f(\omega)|\omega\rangle,
$$

and the frequency distribution $|f(\omega)|^{2}$ is controlled via the tilting angle of the Fabry-Pérot cavity. For any fixed amplitude $f(\omega)$, the dynamics of polarization can be described by a family of CPT maps $\{\Lambda(t)\}_{t \geqslant 0}$ such that

$$
\Lambda(t)\left(\begin{array}{ll}
\rho_{H H} & \rho_{H V} \\
\rho_{V H} & \rho_{V V}
\end{array}\right)=\left(\begin{array}{lc}
\rho_{H H} & k^{*}(t) \rho_{H V} \\
k(t) \rho_{V H} & \rho_{V V}
\end{array}\right),
$$

where $\rho_{\lambda \lambda^{\prime}}=\left\langle\lambda\left|\rho_{S}(0)\right| \lambda^{\prime}\right\rangle\left(\lambda, \lambda^{\prime}=H, V\right)$ with $\rho_{S}(0)$ the initial polarization state, and the time-dependent dephasing function

$$
k(t)=\int d \omega|f(\omega)|^{2} e^{i\left(n_{V}-n_{H}\right) \omega t}
$$

has been introduced. Regardless of the choice of $f(\omega)$, the pair of initial system states that maximizes the increase of trace distance is

$$
\left|\psi_{S}^{ \pm}(0)\right\rangle=\frac{1}{\sqrt{2}}(|H\rangle \pm|V\rangle) .
$$

The calculation of the corresponding trace distance at a time $t$ is straightforward and leads us to $\mathcal{N}(\Lambda)=\sum_{j}^{\prime}\left[\left|k\left(b_{j}\right)\right|-\right.$ $\left.\left|k\left(a_{j}\right)\right|\right]$, where the sum is taken over the temporal region of extremes $a_{j}$ and $b_{j} \geqslant a_{j}$ where $|k(t)|$ grows. The dynamics at hand is thus non-Markovian if and only if

$$
\left|k\left(t+t^{\prime}\right)\right|>|k(t)|
$$

for some $t, t^{\prime} \geqslant 0$. By adjusting the distribution $f(\omega)$, one can arrange for a transition from Markovian to non-Markovian open-system dynamics [23], which can be characterized through the time-local master equation

$$
\frac{d}{d t} \rho_{S}(t)=-i \epsilon(t)\left[\sigma_{z}, \rho_{S}(t)\right]+\gamma(t)\left[\sigma_{z} \rho_{S}(t) \sigma_{z}-\rho_{S}(t)\right],
$$

where $\epsilon(t)=-\frac{1}{2} \operatorname{Im}\left[\partial_{t} \ln k(t)\right]$ and $\gamma(t)=-\frac{1}{2} \operatorname{Re}\left[\partial_{t} \ln k(t)\right]$.

Let us now address how our general analysis applies to this model. The transition from Markovian to non-Markovian dynamics is shown by following the evolution of the trace distance for the pair of initial reduced states in Eq. (23) upon variation of the initial state of the environment, as set by $f(\omega)$. Thus, we can focus on the evolution of the initial total states $\left|\psi_{S E}^{1}(0)\right\rangle=\left|\psi_{S}^{+}(0)\right\rangle \otimes\left|\psi_{E}(0)\right\rangle$ and $\left|\psi_{S E}^{2}(0)\right\rangle=$ $\left|\psi_{S}^{-}(0)\right\rangle \otimes\left|\psi_{E}(0)\right\rangle$. The corresponding total states at time $t$ are found using Eq. (19). In turn, this allows us to evaluate analytically all the quantities of interest introduced in Sec. II, which are defined in terms of the trace norm of operators on the Hilbert space of the open system. It turns out that the two environmental states are equal at all times, so that the possible increases of the trace distance can be traced back 
(a)

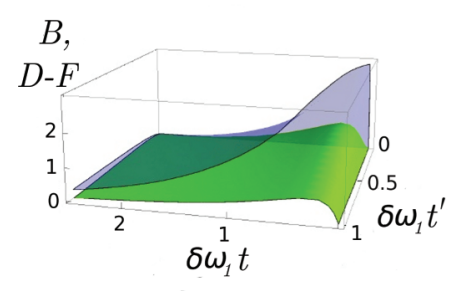

(b)

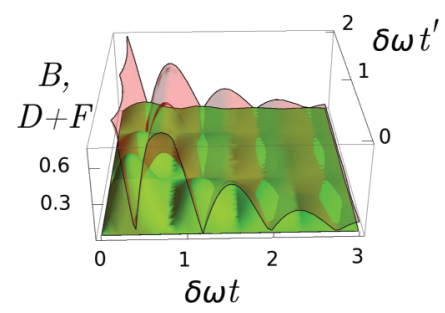

(c)

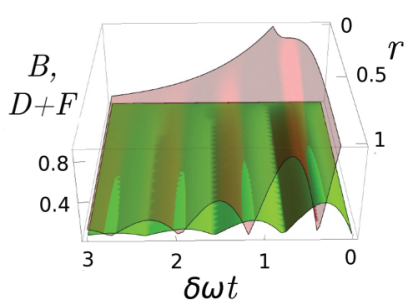

FIG. 2. (Color online) Effects of system-environment correlations described through the function $B\left(t^{\prime}, t^{\prime} \rho^{1,2}\right)$ given in Eq. (26) corresponding to the example discussed in Sec. III A for a frequency distribution as in Eq. (28). (a) We have taken $\omega_{1}^{0} / \delta \omega_{1}=\omega_{2}^{0} / \delta \omega_{1}, \delta \omega_{2} / \delta \omega_{1}=10$, and $r=1$. In both (b) and (c) we have taken $\delta \omega_{1,2} \equiv \delta \omega, \omega_{1}^{0} / \delta \omega=1$, and $\omega_{2}^{0} / \delta \omega=9$ with $r=1\left(t^{\prime} \delta \omega=0.3\right)$ in (b) [(c)]. Semitransparent surfaces represent $D\left(t, \rho^{1,2}\right)-F\left(t^{\prime}, t, \rho^{1,2}\right)$ (a) and $D\left(t, \rho^{1,2}\right)+F\left(t^{\prime}, t, \rho^{1,2}\right)[$ (b) and (c)].

solely to the correlations built during the interaction between the open system and the environment. In particular, we find that the effects of system-environment correlations at time $t$ on the subsequent evolution of the open system are quantified through

$$
B\left(t^{\prime}, t, \rho^{1,2}\right)=\left|k\left(t+t^{\prime}\right)-k(t) k\left(t^{\prime}\right)\right| .
$$

Moreover, we have that $F\left(t^{\prime}, t, \rho^{1,2}\right)=\left|k(t) k\left(t^{\prime}\right)\right|$ and $\Delta D\left(t^{\prime}, t, \rho^{1,2}\right)=\left|k\left(t+t^{\prime}\right)\right|-|k(t)|$. Therefore, Eq. (10) is indeed satisfied. These quantities can be explicitly evaluated by specifying the frequency distribution $|f(\omega)|^{2}$ that determines $k(t)$ through Eq. (22). As a first example, we use a Lorentzian distribution: as we shall see, this leads to a semigroup evolution and thus provides a natural benchmark. Explicitly, we take

$$
|f(\omega)|^{2}=\frac{\delta \omega}{\pi\left[\left(\omega-\omega^{0}\right)^{2}+\delta \omega^{2}\right]},
$$

where $\omega^{0}$ is the central frequency and $\delta \omega$ is the width of the Lorentzian distribution, which can be obtained using single photons emitted by quantum dots [24]. Choosing a Lorentzian frequency distribution corresponds to taking $k(t)=$ $e^{\left(i \omega_{0}-\delta \omega\right) t}$, which entails the exponential decay of the trace distance $D\left(t, \rho^{1,2}\right)=e^{-\delta \omega t}$. In this case, the coefficients of the time-local generator in Eq. (25) reduce to $\epsilon(t)=\omega_{0} / 2$ and $\gamma(t)=\delta \omega / 2$, so that the dynamics of the open system is fixed by a completely positive semigroup [41,42]. In addition, the exponential expression of $k(t)$ means that the correlations between the open system and the environment have no influence on the evolution of the trace distance between reduced states, as follows from Eq. (26). Thus, for the model at hand, in the semigroup regime systemenvironment correlations, despite being present, do not affect the dynamics of the open system at all. The exponential expression for $k(t)$ leads to $B\left(t^{\prime}, t, \rho^{1,2}\right)=0$, which in turn implies $D\left(t^{\prime}+t, \rho^{1,2}\right)=F\left(t^{\prime}, t, \rho^{1,2}\right)$ : States $\rho_{S E}^{j}(t)(j=1,2)$ can be replaced with $\rho_{S}^{j}(t) \otimes \rho_{E}(t)$ without modifying the subsequent evolution of the trace distance.

Let us now consider the frequency distribution

$$
|f(\omega)|^{2}=\sum_{j=1,2} \frac{A_{j} \delta \omega_{j}}{\left(\omega-\omega_{j}^{0}\right)^{2}+\delta \omega_{j}^{2}},
$$

so that $k(t)=\left[e^{\left(i \omega_{1}^{0}-\delta \omega_{1}\right) t}+r e^{\left(i \omega_{2}^{0}-\delta \omega_{2}\right) t}\right] /(1+r)$, with $r=$ $A_{2} / A_{1}$. As can be easily checked, if the two Lorentzian distributions in Eq. (28) have the same central frequencies, $\omega_{1}^{0}=\omega_{2}^{0}$, the resulting dynamics is still Markovian as $|k(t)|$ is monotonically decreasing. Incidentally, differently from the case of a single Lorentzian distribution, the family of CPT maps determined by Eq. (21) is no longer a semigroup but a divisible family of completely positive dynamical maps [21]. In fact, the coefficients of the corresponding generator in Eq. (25) are given by

$$
\epsilon(t)=\frac{\omega_{0}}{2}, \quad \gamma(t)=\frac{\delta \omega_{1} e^{-\delta \omega_{1} t}+r \delta \omega_{2} e^{-\delta \omega_{2} t}}{2\left(e^{-\delta \omega_{1} t}+r e^{-\delta \omega_{2} t}\right)},
$$

which are positive at all times. Our analysis allows the Markovianity of the dynamics to be traced back to the weakness of the system-environment correlations created by the interaction. In Fig. 2(a), we plot $B\left(t^{\prime}, t, \rho^{1,2}\right)$ and $D\left(t, \rho^{1,2}\right)-F\left(t^{\prime}, t, \rho^{1,2}\right)$. Both the quantities refer to the two initial states of the system in Eq. (23) and are plotted as functions of $t$ and $t^{\prime}$. The effects of system-environment correlations on the reduced system, as quantified through $B\left(t^{\prime}, t, \rho^{1,2}\right)$, are always weaker than the threshold embodied by $D\left(t, \rho^{1,2}\right)-F\left(t^{\prime}, t, \rho^{1,2}\right)$. As a consequence, the distinguishability between the states of the open system cannot increase and a Markovian dynamics is induced.

A different situation occurs if the two Lorentzian distributions in Eq. (28) have the same width but different central frequencies. In this case, the dynamics is non-Markovian, i.e., $|k(t)|$ is a nonmonotonic function of time. As shown in Fig. 2(b), the effects of system-environment correlations are now stronger than the upper threshold $D\left(t, \rho^{1,2}\right)+$ $F\left(t^{\prime}, t, \rho^{1,2}\right)$. Basically, there are times $t$ such that the two reduced states, $\rho_{S}^{1}(t)$ and $\rho_{S}^{2}(t)$ are very similar, so that the upper threshold $D\left(t, \rho^{1,2}\right)+F\left(t^{\prime}, t, \rho^{1,2}\right)$ is small, while the correlations of the two total states, $\chi_{S E}^{1}(t)$ and $\chi_{S E}^{2}(t)$, are still different. Consequently, the trace distance at subsequent times $t+t^{\prime}$ increases. In this case, system-environment correlations do induce a non-Markovian dynamics. Finally, by taking $r$ in Eq. (28) from zero to any nonzero value, we have a transition from a Markovian dynamics, more precisely a semigroup dynamics, to a non-Markovian dynamics. In Fig. 2(c) one can see how this is reflected in the behavior of $B\left(t^{\prime}, t, \rho^{1,2}\right)$. In fact, the latter turns from being identically zero for $r=0$ to increasing above the upper threshold $D\left(t, \rho^{1,2}\right)+F\left(t^{\prime}, t, \rho^{1,2}\right)$, thus implying a non-Markovian dynamics. 
(a)

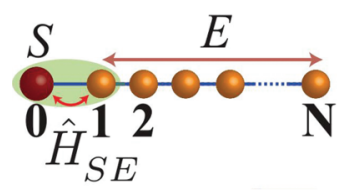

(b)

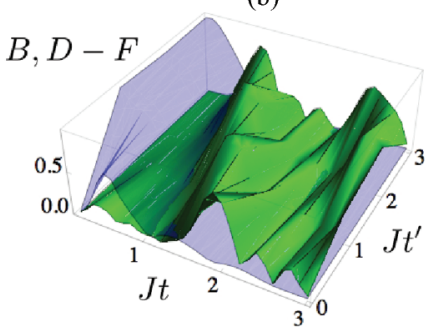

(c)

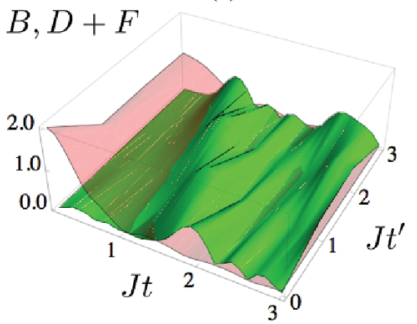

FIG. 3. (Color online) (a) We consider a quantum spin chain of $N+1$ particles partitioned into a two-level-system spin embodied by particle 0 and a finite-size environment (provided by the rest of the chain). The dynamics experienced by spin 0 can be changed from Markovian to strongly non-Markovian by adjusting the parameters entering the system-environment coupling model $\hat{H}_{S E}$ and the interenvironment one $\hat{H}_{E}$. Details of the form of such Hamiltonians are given in the body of the paper. (b) [(c)] Comparison between the function $B\left(t^{\prime}, t, \rho^{1,2}\right)($ full-color surface) and the threshold for Markovianity (non-Markovianity) $D\left(t, \rho^{1,2}\right)-F\left(t^{\prime}, t, \rho^{1,2}\right)\left[D\left(t, \rho^{1,2}\right)+F\left(t^{\prime}, t, \rho^{1,2}\right)\right]$. (semitransparent curves). We have used $N=8$ with $J_{0} / J=1$ and $B / J=10^{-2}$.

\section{B. Non-Markovianity in a spin-chain system}

As a second instance, we consider the case of a single qubit attached to a quantum spin chain of $N$ spin-1/2 particles, along the lines of the studies reported in Refs. [9,35] and as illustrated in Fig. 3(a). The open system is embodied by spin 0 , while the environment consists of particles $1 \rightarrow N$. The overall $N+1$ system is mutually coupled via an $X X$ model and subjected to a transverse magnetic field. Assuming units such that $\hbar=1$, the corresponding Hamiltonian model is $\hat{H}=\hat{H}_{S E}+\hat{H}_{E}$ with

$$
\begin{aligned}
\hat{H}_{S E} & =-2 J_{0}\left(\hat{\sigma}_{0}^{x} \hat{\sigma}_{1}^{x}+\hat{\sigma}_{0}^{y} \hat{\sigma}_{1}^{y}\right), \\
\hat{H}_{E} & =-2 J \sum_{n=1}^{N-1}\left(\hat{\sigma}_{n}^{x} \hat{\sigma}_{n+1}^{x}+\hat{\sigma}_{n}^{y} \hat{\sigma}_{n+1}^{y}\right)-2 B \sum_{n=1}^{N} \hat{\sigma}_{n}^{z},
\end{aligned}
$$

where $\hat{\sigma}_{n}^{k}$ is the $k$ Pauli matrix $(k=x, y, z)$ for particle $n, B$ is the amplitude of the magnetic field affecting $S$, and $J\left(J_{0}\right)$ is the interenvironment (system-environment) coupling strength. The underlying assumption is that the free evolutions of $S$ and $E$ are identical, thus allowing the passage to the interaction picture without the introduction of time-dependent coefficients. The non-Markovian evolution experienced by spin 0 was characterized fully in Ref. [9], where it was found that, for interaction times that are within the recurrence time of the system (when any information propagating across the chain returns to the open system after reaching the end of the chain), there is a working point defined by $\left(J_{0} / J, B / J\right)$ at which the measure of non-Markovianity $\mathcal{N}$ is null. As the optimization inherent in the definition of such a measure is achieved for system states lying on the equatorial plane of the Bloch sphere [9], we consider the input states $\rho_{S}^{ \pm}(0)=\left|\psi_{S}^{ \pm}(0)\right\rangle\left\langle\psi_{S}^{ \pm}(0)\right|$, while the environment is initialized in $\rho_{1,2}^{E}(0)=\rho_{\text {ini }}^{E}=\otimes_{i=1}^{N-1}|0\rangle_{i}\langle 0|$. In order to provide a physically significant example that is nevertheless able to show clearly the features that we are interested in, we solved fully the problem embodied by an environment of $N=8$ spins with $J_{0} / J=1$ and $B / J=10^{-2}$. At variance with the previous example, the system lacks an analytically amenable solution (the expressions for the trace distance and the quantities introduced in Sec. II are too involved to be reported here) but allows for a handy numerical analysis. The results are shown in Figs. 3(b) and 3(c), where $B\left(t, t^{\prime}, \rho^{1,2}\right)$ is compared to the thresholds $D\left(t, \rho^{1,2}\right) \pm F\left(t, t^{\prime}, \rho^{1,2}\right)$ within a broad range of values for $t$ and $t^{\prime}$. Clearly, besides the existence of a range of values of $\left(t, t^{\prime}\right)$ where the dynamics is Markovian as expected, $B\left(t, t^{\prime}, \rho^{1,2}\right)$ soon passes the thresholds for non-Markovianity [cf. Fig. 3(c)]. In particular, this is the case for values of $\left(t, t^{\prime}\right)$ such that $J\left(t+t^{\prime}\right)<3$, which guarantee that the corresponding evolutions occur well within the recurrence times of the spin chain and any non-Markovian effect is due to the intrinsic features of the interaction rather than the finiteness of the environment. Interestingly enough, the gap between the lower and upper thresholds identified above may disappear, in this example. Values of $t$ exist at which $D\left(t, \rho^{1,2}\right) \simeq F\left(t, t^{\prime}, \rho^{1,2}\right)$, thus making the gap between upper and lower thresholds effectively null.

\section{CONCLUSIONS}

We have shown how the dynamical correlations between an open quantum system and its environment influence the nature of the open system's dynamics, as far as non-Markovianity is concerned. Our analysis relies on the definition of nonMarkovianity given in terms of the evolution of the trace distance between reduced states [20].

We have introduced the quantity $F\left(t^{\prime}, t, \rho^{1,2}\right)$ [cf. Eq. (4)] that describes the evolution of the trace distance under the CPT maps that would connect states at different times if correlations and environmental changes could be neglected at any time. In a complementary way, we have defined the quantity $B\left(t^{\prime}, t, \rho^{1,2}\right)$ [see Eq. (5)] that measures, by means of the trace norm, the effects of system-environment correlations and environmental evolution due to the interaction up to a time $t$ on the subsequent dynamics of the open system. These two quantities allow introduction of an upper and a lower bound to the variation of the trace distance between reduced states on finite time intervals, as quantified by Eq. (10). We have thus been able to conclude that if the effects of correlations and environmental evolution are below a first threshold the resulting reduced dynamics is certainly Markovian. Despite being necessary, system-environment correlations and changes in the environment are not a priori sufficient to induce an increase of the trace distance. On the other hand, if their effects exceed a second threshold, a non-Markovian reduced dynamics is surely induced. 
The general analysis has been applied to the model exploited in Ref. [23] to experimentally detect the transition between Markovian and non-Markovian dynamics. We have shown how such a transition can be explained in terms of the different effects of system-environment correlations. By properly varying the initial state of the environment, one can describe both a semigroup dynamics, in which systemenvironment correlations do not affect at all the evolution of the reduced state's distinguishability, and non-Markovian dynamics, in which system-environment correlations strongly influence the dynamics of the open system.

Our results will be useful also with respect to different approaches to non-Markovianity relying on other properties of the dynamical maps, since they show in full generality to what extent system-environment correlations can be compatible with a contraction of the trace distance. In particular, this could help to further understand the connection between correlations in the total state and breaking of divisibility of the completely positive dynamical maps [5,12]. In addition, our results could provide further insights into microscopic derivations of reduced dynamics, in order to clarify the role of system-environment correlations and changes in the environmental state [43].

\section{ACKNOWLEDGMENTS}

A.S. and B.V. acknowledge financial support from COST Action MP1006. L.M. is supported by the EU through a Marie Curie IEF Fellowship. M.P. thanks the UK EPSRC for the support of a Career Acceleration Fellowship and a grant of the New Directions for Research Leaders initiative (No. EP/G004579/1).
[1] H.-P. Breuer and F. Petruccione, The Theory of Open Quantum Systems (Oxford University Press, Oxford, 2002).

[2] A. A. Budini, Phys. Rev.A 69, 042107 (2004).

[3] J. Piilo, S. Maniscalco, K. Härkönen, and K.-A. Suominen, Phys. Rev. Lett. 100, 180402 (2008).

[4] H.-P. Breuer and B. Vacchini, Phys. Rev. Lett. 101, 140402 (2008); Phys. Rev. E 79, 041147 (2009).

[5] A. Shabani and D. A. Lidar, Phys. Rev. Lett. 102, 100402 (2009).

[6] L. Mazzola, S. Maniscalco, J. Piilo, K.-A. Suominen, and B. M. Garraway, Phys. Rev. A 80, 012104 (2009).

[7] D. Chruscinski and A. Kossakowski, Phys. Rev. Lett. 104, 070406 (2010).

[8] A. Barchielli, C. Pellegrini, and F. Petruccione, Europhys. Lett 91, 24001 (2010); Phys. Rev. A 86, 063814 (2012).

[9] T. J. G. Apollaro, C. Di Franco, F. Plastina, and M. Paternostro, Phys. Rev. A 83, 032103 (2011).

[10] M. Žnidarič, C. Pineda, and I. García-Mata, Phys. Rev. Lett. 107, 080404 (2011).

[11] B. Vacchini, A. Smirne, E.-M. Laine, J. Piilo, and H.-P. Breuer, New. J. Phys. 13, 093004 (2011); B. Vacchini, J. Phys. B 45, 154007 (2012); A. Smirne, A. Stabile, and B. Vacchini, Phys. Scr. T 153, 014057 (2013).

[12] C. A. Rodríguez-Rosario and E. C. G. Sudarshan, Int. J. Quantum Inf. 9, 1617 (2011).

[13] A. R. Usha Devi, A. K. Rajagopal, S. Shenoy, and R. W. Rendell, J. Quantum Inf. Sci. 2, 47 (2012).

[14] P. Haikka, J. Goold, S. McEndoo, F. Plastina, and S. Maniscalco, Phys. Rev. A 85, 060101(R) (2012).

[15] E.-M. Laine, H.-P. Breuer, J. Piilo, C.-F. Li, and G.-C. Guo, Phys. Rev. Lett. 108, 210402 (2012).

[16] L. Mazzola, C. A. Rodríguez-Rosario, K. Modi, and M. Paternostro, Phys. Rev. A 86, 010102(R) (2012).

[17] C. A. Rodríguez-Rosario, K. Modi, L. Mazzola, and A. AspuruGuzik, Europhys. Lett. 99, 20010 (2012).

[18] W. M. Zhang, P. Y. Lo, H. N. Xiong, Matisse Wei-Yuan Tu, and F. Nori, Phys. Rev. Lett 109, 170402 (2012).

[19] M. M. Wolf, J. Eisert, T. S. Cubitt, and J. I. Cirac, Phys. Rev. Lett. 101, 150402 (2008).
[20] H.-P. Breuer, E.-M. Laine, and J. Piilo, Phys. Rev. Lett. 103, 210401 (2009); E.-M. Laine, J. Piilo, and H.-P. Breuer, Phys. Rev. A 81, 062115 (2010).

[21] Á. Rivas, S. F. Huelga, and M. B. Plenio, Phys. Rev. Lett. 105, 050403 (2010).

[22] S. C. Hou, X. X. Yi, S. X. Yu, and C. H. Oh, Phys. Rev. A 83, 062115 (2011).

[23] B. H. Liu, L. Li, Y.-F. Huang, C.-F. Li, G.-C. Guo, E.-M. Laine, H.-P. Breuer, and J. Piilo, Nat. Phys. 7, 931 (2011).

[24] J.-S. Tang, C.-F. Li, Y.-L. Li, X.-B. Zou, G.-C. Guo, H.-P. Breuer, E.-M. Laine, and J. Piilo, Europhys. Lett. 97, 10002 (2012).

[25] A. Chiuri, C. Greganti, L. Mazzola, M. Paternostro, and P. Mataloni, Sci. Rep. 2, 968 (2012).

[26] B. H. Liu, D.-Y. Cao, Y.-F. Huang, C.-F. Li, G.-C. Guo, H.-P. Breuer, E.-M. Laine, and J. Piilo, Sci. Rep. 3, 1781 (2013).

[27] C. W. Gardiner and P. Zoller, Quantum Noise: A Handbook of Markovian and Non-Markovian Quantum Stochastic Methods with Applications to Quantum Optics (Springer-Verlag, Berlin, 2000).

[28] M. Lax, Phys. Rev. 172, 350 (1968).

[29] S. Swain, J. Phys. A 14, 2577 (1981).

[30] P. Talkner, Ann. Phys. (NY) 167, 390 (1986).

[31] G. W. Ford and R. F. O'Connell, Phys. Rev. Lett. 77, 798 (1996).

[32] M. A. Nielsen and I. L. Chuang, Quantum Computation and Quantum Information (Cambridge University Press, Cambridge, 2000).

[33] E.-M. Laine, J. Piilo, and H.-P. Breuer, Europhys. Lett. 92, 60010 (2010).

[34] H.-P. Breuer, J. Phys. B 45, 154001 (2012).

[35] T. J. G. Apollaro, A. Cuccoli, C. Di Franco, M. Paternostro, F. Plastina, and P. Verrucchi, New J. Phys. 8, 083046 (2010); S. Campbell, T. J. G. Apollaro, C. Di Franco, L. Banchi, A. Cuccoli, R. Vaia, F. Plastina, and M. Paternostro, Phys. Rev. A 84, 052316 (2011).

[36] P. Štelmachovič and V. Bužek, Phys. Rev. A 64, 062106 (2001).

[37] M. B. Ruskai, Rev. Math. Phys. 6, 1147 (1994). 
[38] A. Pernice and W. T. Strunz, Phys. Rev. A 84, 062121 (2011); A. Pernice, J. Helmand, and W. T. Strunz, J. Phys. B 45, 154005 (2012).

[39] A. Brodutch, A. Datta, K. Modi, A. Rivas, and C. A. Rodríguez-Rosario, Phys. Rev. A 87, 042301 (2013).
[40] M. Gessner and H.-P. Breuer, Phys. Rev. Lett. 107, 180402 (2011); Phys. Rev. A 87, 042107 (2013).

[41] G. Lindblad, Commun. Math. Phys. 48, 119 (1976).

[42] V. Gorini, A. Kossakowski, and E. C. G. Sudarshan, J. Math. Phys. 17, 821 (1976).

[43] S. Possanner and B. A. Stickler, Phys. Rev. A 85, 062115 (2012). 